\title{
Renal Transplantation With Expanded Criteria Donors: The Experience of One Portuguese Center
}

\author{
F. Ferrer, A. Mota, R. Alves, C. Bastos, F. Macário, A. Figueiredo, L. Santos, A. Roseiro, B. Parada, \\ J. Pratas, P. Nunes, and M. Campos
}

\begin{abstract}
Background. The shortage of kidneys available for transplantation has led to enlarged criteria donors (ECD): namely, donors older than 60 years or aged between 50 and 59 years with 2 of the following characteristics-hypertension, predonation serum creatinine level higher than $1.5 \mathrm{mg} / \mathrm{dL}$ or cerebrovascular disease as the cause of death. The aim of this study was to analyze renal transplants using ECD compared with standard criteria donors (SCD) concerning the incidences of delayed graft function (DGF), acute rejection episodes (ARE), and patient and graft survivals.
\end{abstract}

Materials and Methods. This retrospective study of 409 cadaveric renal transplants over the last 4 years identified ECD in contrast with SCD.

Results. Of the transplants, $24.4 \%$ used ECD. The baseline characteristics of recipients of ECD versus SCD kidneys were similar, except for age and cold ischemia time. Comparing ECD and SCD, we observed an higher incidence of DGF (35\% vs 18\%), occurrence of ARE (34.4\% vs 16.6\%), average serum creatinine levels at 6 (1.87 vs 1.4 $\mathrm{mg} / \mathrm{dL}$ ), and 12 months (1.88 vs $1.43 \mathrm{mg} / \mathrm{dL})$ as well as lower graft survival at 1 (82\% vs $91 \%$ ) and 3 years (75\% vs $84 \%$ ) after transplantation. Recipient survival at 1 year was not different. Multivariate analysis identified recipient age, cold ischemia time, ARE, and DGF as risk factors for graft failure.

Conclusions. Renal transplantation with grafts from ECD shows significantly worse outcomes with higher rates of DGF and ARE, worse graft function, and lower graft survival.

$\mathrm{T}$ HE RISING prevalence of chronic kidney disease (CKD) worldwide has dramatically increased the number of patients who start renal replacement therapy (RRT). ${ }^{1}$ Kidney transplantation is the preferred RRT; it is superior to dialysis in terms of quality of life and long-term mortality risk. ${ }^{2,3}$ In an effort to address the increasing disparity between organ supply and demand, various strategies have been employed to expand donor selection criteria, to include those who might have been deemed unsuitable in earlier times. In 2002, the term expanded criteria donors (ECD) was codified to be brain-dead donors older than 60 years or those aged between 50 and 59 years with 2 of the following characteristics: history of hypertension, terminal predonation serum creatinine level higher than 1.5 $\mathrm{mg} / \mathrm{dL}(132.6 \mu \mathrm{mol} / \mathrm{L})$ and cerebrovascular disease (CVD) as the cause of death. ${ }^{4}$ In addition, donors who were diabetic or non-heartbeating or yielding organs with pro- longed cold preservation time have been characterized to be of marginal quality or ECD. ${ }^{5-9}$

Allograft outcomes with ECD/marginal kidneys are, in most series, inferior to that of organs considered to be from ideal or standard criteria donors (SCD), namely, kidneys from a reference group of normotensive donors, aged between 10 and 39 years, with preretrieval serum creatinine concentrations lower than $1.5 \mathrm{mg} / \mathrm{dL}$ and deaths from causes other than stroke. ${ }^{10,11}$ Indeed, the risk of graft failure after a transplantation with a marginal kidney is

From the Renal Transplantation Unit, Department of Urology and Renal Transplantation, Hospitais da Universidade de Coimbra, Portugal.

Address reprint requests to F. Ferrer, Renal Transplantation Unit, Hospitais da Universidade de Coimbra, 3000-075 Coimbra, Portugal. E-mail: franciscodina@gmail.com

0041-1345/09/\$-see front matter doi:10.1016/j.transproceed.2009.02.044 
about $70 \%$ higher than with a non-ECD transplant. ${ }^{4}$ Notwithstanding the diminished survival of these ECD kidneys, renal transplantation significantly improves survival and quality of life when compared with those among patients on regular dialysis, who remain on waiting lists. ${ }^{12}$ The aims of this study were to report our experience with ECD versus SCD kidney transplants, with a special emphasis on the incidence of delayed graft function (DGF), acute rejection episodes (ARE) as well as graft and patient survival.

\section{MATERIALS AND METHODS}

This work examines a historical cohort of 409 cadaver renal transplants performed by us over the last 4 years. ECD kidneys were selected according to age ( $>60$ years) and cause of death (CVD). For all recipients of cadaveric renal transplants, we obtained the following data: demographic information, age, gender, weight $(\mathrm{kg})$, time on dialysis, comorbidities, HLA compatibilities, cold ischemia time, immunosuppression at the time of transplantation, AREs, DGF, serum creatinine at 1 and 3 years posttransplantation, as well as graft and recipient survival at 1 and 3 years posttransplantation. For all donors, we evaluated age, gender, comorbidities (arterial hypertension, diabetes, and atherosclerosis), preretrieval serum creatinine and cause of death.

The 409 patients were divided into 2 groups according to the kind of donor: group 1 (with a 100 recipients of ECD) and group 2 (with 309 recipients, of SCD). The statistical analysis used the Student $t$ test for continuous variables and the $\chi^{2}$ test for categorical variables. Recipient and graft survivals were evaluated by the Kaplan-Meier method. The most relevant factors associated with graft outcome were analyzed using a multivariate Cox regression proportional hazards model. A $P$ value less than .05 was considered significant.

\section{RESULTS}

The prevalence of kidney transplants from ECD was $24.4 \%$ (100/409). When compared with SCD, the ECD were older $(60.46 \pm 11.99$ vs $39.05 \pm 14.36$ years; $P=.000)$ and had a greater frequency of death owing to CVD (70 vs $39.6 \% ; P=$ $.000)$.

Table 1 shows the main features of the recipients. The ECD kidney recipients were also older $(52.48 \pm 11.93$ vs $44.44 \pm 13.01$ years) and showed a higher cold ischemia time $(19.72 \pm 5.61$ vs $17.81 \pm 5.58)$. No differences were detected between the groups with respect to gender, weight, time, dialysis modality, comorbidities, or number of HLA compatibilities. Immunosuppression was based on calcineurin inhibitors in $79 \%$ of ECD kidney transplants and $78 \%$ of SCD. Forty-seven percent of ECD kidney recipients and $55 \%$ of SCD kidney recipients received induction with antibodies (thymoglobulin or basiliximab), although the difference did not attain statistical significance $(P=.200)$.

The incidence of AREs was significantly greater among recipients of ECD (34.4\% vs $16.6 \%)$, and there was a higher incidence of DGF (34.7\% vs $18 \%$ ). Serum creatinine values were also higher at 6 and 12 months among ECD recipients. Graft survivals at 1 and at 3 years were lower among ECD kidney recipients, although patient survivals at 1 and 3 years were similar in both groups (Table 1).
Table 1. Recipient Characteristics

\begin{tabular}{lccc}
\hline & ECD $(n=100)$ & SCD $(n=309)$ & $P$ \\
\hline Age (y) & $52.48 \pm 11.93$ & $44.44 \pm 13.01$ & .000 \\
Female gender (\%) & $29 \%$ & $36 \%$ & .200 \\
Weight (kg) & $66.16 \pm 10.57$ & $63.73 \pm 13.84$ & .066 \\
Time on dialysis (mo) & $55.99 \pm 43.58$ & $51.85 \pm 48.22$ & .423 \\
Comorbidities (\%) & $79.8 \%$ & $75 \%$ & .406 \\
Cold ischemia time (h) & $19.72 \pm 5.61$ & $17.81 \pm 5.59$ & .003 \\
HLA compatibilities & $1.53 \pm 0.50$ & $1.50 \pm 0.50$ & .550 \\
Induction with antibodies (\%) & $47 \%$ & $55 \%$ & .200 \\
AREs (\%) & $34.4 \%$ & $16.6 \%$ & .000 \\
DGF (\%) & $34.7 \%$ & $18 \%$ & .001 \\
Cr at 6 mos (mg/dL) & $1.87 \pm 0.80$ & $1.41 \pm 0.50$ & .000 \\
Cr at 12 mos (mg/dL) & $1.89 \pm 0.75$ & $1.43 \pm 0.58$ & .000 \\
Graft survival at 1 y (\%) & $82 \%$ & $91 \%$ & .029 \\
Graft survival at 3 y (\%) & $75 \%$ & $84 \%$ & .003 \\
Recipient survival at 1 y (\%) & $94 \%$ & $95 \%$ & .793 \\
Recipient survival at 3 y (\%) & $88 \%$ & $89 \%$ & .793 \\
\hline \multicolumn{2}{c}{ Abbreviations: ARE, acute rejection episode; Cr, creatinine; DGF, delayed } \\
graft function; ECD, extended criteria donor; SCD, standard criteria donor.
\end{tabular}

Multivariate analysis of potential determinants of graft survival revealed that recipient age $(P<.05)$, cold ischemia time $(P<.05)$, and incidences of AREs and DGF $(P<.05)$ were independently associated with graft failure (Table 2$)$.

\section{DISCUSSION}

This study demonstrated that renal transplants using ECD grafts displayed significantly worse results. The incidences of DGF and AREs were higher among these transplants. Graft function estimates (measured by serum creatinine at 6 and 12 months) as well as graft survivals at 1 and 3 years were also worse among ECD recipients.

Although ECD kidneys are mainly used in older recipients, who display a variety of comorbidities, this practice did not solely explain the worse results; indeed, it should be considered safe because older recipients are less liable to experience AREs and somewhat lower kidney function. ${ }^{13}$ In fact, some studies have shown comparable graft survivals between ECD and SCD kidneys ${ }^{9,13}$; outcome differences have been partially explained by various definitions of ECD. In our study, the longer cold ischemia time as well as the higher frequencies of AREs and DGF observed in ECD transplants may further reduce residual renal function and may have also contributed to the observed reduced graft function/survival. Our multivariate analysis of the factors associated with graft survival confirmed that, along with recipient age, these were the most significant determinants of graft outcomes. Indeed, transplanting kidneys with a reduced nephron mass, according to age and associated vascular disease or diabetes, makes them more sensitive to the cold ischemia time and AREs. ${ }^{14}$ Therefore, all efforts must be directed to diminish the cold ischemia time for these particular kidneys, to avoid other, nonimmunologic risk factors such as nephrotoxic therapies and to enhance the "old for old" program. 
Table 2. Multivariate Cox Regression Analysis of Factors Potentially Associated With Graft Failure

\begin{tabular}{lccc}
\hline & $P$ & Hazard Ratio & $95 \% \mathrm{Cl}$ \\
\hline Age of recipient (y) & .045 & 1.892 & $1.013-3.531^{*}$ \\
Time on dialysis (mos) & .447 & 0.791 & $0.432-1.447^{\dagger}$ \\
Comorbidities & .051 & 1.712 & $0.997-4.062^{\ddagger}$ \\
Cold ischemia time (h) & .047 & 1.787 & $1.009-3.164^{q}$ \\
HLA compatibilities & .504 & 1.205 & $0.697-2.081^{\S}$ \\
Induction with antibodies & .510 & 1.199 & $0.698-2.060^{\ddagger}$ \\
Acute rejection episode & .005 & 2.261 & $1.277-4.002^{\ddagger}$ \\
Delayed graft function & .000 & 2.655 & $1.545-4.651^{\ddagger}$ \\
Donor & .588 & 1.190 & $0.634-2.233^{\|}$
\end{tabular}

$\mathrm{Cl}$, confidence interval.

${ }^{*}<60$ versus $\geq 60$ years

$\dagger<60$ versus $\geq 60$ months.

$\ddagger$ Yes versus no.

१ $<24$ versus $\geq 24$ hours.

$\S<3$ versus $\geq 3$.

"ECD versus SCD.

In accordance with other studies, 9,13 our analysis showed that patient survivals at the end of the first and third years posttransplantation were comparable between ECD and SCD kidney recipients, and probably superior to those of matched patients on regular dialysis program, who remained on the waiting list. ${ }^{12}$ For this reason, the use of ECD kidneys is well justified owing to the dramatic shortage of available organs for transplantation because it increases the donor pool and possibly patient life expectancy.

\section{REFERENCES}

1. U.S. Renal Data System: USRDS 2006 annual data report: Atlas of end-stage renal disease in the United States, National Institutes of Health, National Institute of Diabetes and Digestive and Kidney Diseases, Bethesda, MD. Am J Kidney Dis 47(suppl 1):S1, 2006
2. Wolfe RA, Ashby VB, Milford EL, et al: Comparison of mortality in all patients on dialysis, patients on dialysis awaiting transplantation, and recipients of a first cadaveric transplant. N Engl J Med 341:1725, 1999

3. Port FK, Wolfe RA, Mauger EA, et al: Comparison of survival probabilities for dialysis patients vs cadaveric renal transplant recipients. JAMA 270:1339, 1993

4. Port FK, Bragg-Gresham JL, Metzger RA, et al: Donor characteristics associated with reduced graft survival: an approach to expanding the pool of kidney donors. Transplantation 74:1281, 2002

5. Tullius SG, Reutzel-Selke A, Nieminen-Kelha M, et al: Contribution of donor age and ischemic injury in chronic renal allograft dysfunction. Transplant Proc 31:1298, 1999

6. Remuzzi G, Grinyò J, Ruggenenti P, et al: Early experience with dual kidney transplantation in adults using expanded donor criteria. J Am Soc Nephrol 10:2591, 1999

7. Cho YW, Cecka JM, Gjertson DW, et al: Prolonged hypertension ( $>10$ years) is a significant risk factor in older cadaver donor renal transplants. Transplant Proc 31:1283, 1999

8. Shenoy S, Lowell JA, Flye MW, et al: Use of extended donors in high-risk renal transplant recipients: a 2-year single-center experience. Transplant Proc 28:95, 1996

9. Keitel E, Michelon T, Santos AF, et al: Renal transplants using expanded cadaver donor criteria. Ann Transplant 9:23, 2004

10. Golconda MS, Whiting JF, Smith R, et al: Long-term outcome of kidney transplantation from expanded criteria donors: a single center experience. Transplant Proc 29:3379, 1997

11. Sung RS, Christensen LL, Leichtman AB, et al: Determinants of discard of expanded criteria donor kidneys: impact of biopsy and machine perfusion. Am J Transplant 8:783, 2008

12. Merion RM, Ashby VB, Wolfe RA, et al: Deceased-donor characteristics and the survival benefit of kidney transplantation. JAMA 294:2726, 2005

13. Stratta RJ, Rohr MS, Sundberg AK, et al: Increased kidney transplantation utilizing expanded criteria deceased organ donors with results comparable to standard criteria donor transplant. Ann Surg 239:688, 2004

14. Frutos MA, Sola E, Mansilla P, et al: Expanded criteria donors for kidney transplantation: quality control and results. Trasnplant Proc 38:2371, 2006 